\title{
Navigating the Wrench-Feasible C-Space of Cable-Driven Hexapods
}

Oriol Bohigas, Montserrat Manubens, and Lluís Ros

\begin{abstract}
Motion paths of cable-driven hexapods must carefully be planned to ensure that the lengths and tensions of all cables remain within acceptable limits, for a given wrench applied to the platform. The cables cannot go slack -to keep the control of the platform- nor excessively tight -to prevent cable breakage- even in the presence of bounded perturbations of the wrench. This paper proposes a path planning method that accommodates such constraints simultaneously. Given two configurations of the platform, the method attempts to connect them through a path that, at any point, allows the cables to counteract any wrench lying inside a predefined uncertainty region. The resulting $\mathrm{C}$-space is placed in correspondence with a smooth manifold, which allows defining a continuation strategy to search this space systematically from one configuration, until the second configuration is found, or path non-existence is proved by exhaustion of the search. The approach is illustrated on the NIST Robocrane hexapod, but it remains applicable to general cable-driven hexapods, either to navigate their full six-dimensional C-space, or any of its slices.
\end{abstract}

Key words: Cable-driven hexapod, tendon, wire, higher-dimensional continuation, wrench-feasible C-space, Robocrane.

\section{Introduction}

In recent years, cable-driven parallel mechanisms have been increasingly studied and applied to more and more relevant tasks, such as manipulation of heavy loads [7, 20], high-precision positioning [18], monitoring of aquatic environments [4, 13], automated construction of civil structures [5], rescue systems [19], or motion simulators [27], among others. Their generally simple design, consisting of a moving platform connected to a fixed base by means of cables that wind up on winches, re-

O. Bohigas, M. Manubens, and L. Ros,

Institut de Robòtica i Informàtica Industrial, e-mail: \{obohigas,mmanubens,lros\}@iri.upc.edu. 
sults in manipulators with low weight but high load capacity. These advantages, together with the fact that they can usually achieve larger workspaces than their counterparts with rigid-limb legs, make cable-driven manipulators very energy-efficient and appropriate to maneuver heavy loads. However, additional constraints apply: their cables can pull but are unable to push the platform, which obliges to keep the tensions in all cables positive during normal operation.

The workspace of a cable-driven manipulator is limited by a number of hypersurfaces where the control of the manipulator is compromised [28], corresponding to configurations where the tension of some cable is either zero, for which the cable goes slack and control of one degree of freedom is lost, or goes to infinity, which indicates that the mechanism is on a singular configuration and the cable can brake. In practice, it is important to prevent both extreme situations, and ensure that the cables work within a predefined range of admissible tensions for a given wrench applied on the platform, subject to bounded perturbations in all directions. Several authors have proposed strategies for the determination of wrench-feasible workspaces [6,12, 23, 26, 29, 28], but the problem of planning paths between two configurations in such spaces has not been intensively studied. Some algorithms exist that try to avoid the singular configurations where forces tend to infinity, but they are mainly tailored to classical Stewart platforms with UPS legs [2, 9, 25, 10], and their application to cable-driven manipulators is not straightforward because they do not account for the positivity constraint on the leg tensions. Moreover, these algorithms measure the clearance of the path relative to the singularity locus using the determinant or the condition number of the Jacobian matrix, which, as noted in [30], lack physical significance. While some approaches indeed exist for cabledriven manipulators $[11,15,17]$, the path they compute is evaluated for feasibility at discrete points only, and the fulfillment of all constraints along the whole path is not guaranteed.

This paper provides a method for planning paths on the wrench-feasible C-space of cable-driven hexapods, also called tendon-based Stewart platforms. This C-space is defined following the spirit of [6], as the one that results from only allowing wrench-feasible configurations; i.e., those on which the cable tensions remain within the allowed limits, for any platform wrench belonging to a prescribed sixdimensional region (Section 2). The method relies on defining a system of equations whose solution manifold corresponds to the wrench-feasible C-space of the hexapod, so that maneuvering through such manifold guarantees singularity avoidance at all times, while maintaining cable tensions and lengths within their allowable bounds (Section 3). This manifold, as well as any of its slices obtained by fixing some of the pose parameters, can be proved to be smooth everywhere, which allows defining a systematic higher-dimension continuation strategy to explore the manifold from a start configuration, until a path to a goal configuration is found, or path non-existence is proved (Section 4). The approach has been implemented and validated on several experiments (Section 5), and points for future attention have been identified (Section 6). 


\section{Problem statement}

A cable-driven hexapod consists of a moving platform suspended from a fixed base by means of six cables, winding around independent winches (Fig. 1). When all cables are in tension, which can be achieved by the action of gravity, their lengths determine a locally unique pose for the platform, so that it is possible to control the six degrees of freedom of the platform by actuating the winches.

Let $O X Y Z$ and $P X^{\prime} Y^{\prime} Z^{\prime}$ be fixed and moving reference frames respectively attached to the base and the platform (Fig. 1). Any configuration of the platform can be uniquely represented by a pair $\boldsymbol{q}=(\boldsymbol{p}, \boldsymbol{R}) \in S E(3)$, where $\boldsymbol{p}=[x, y, z]^{\top}$ is the position vector of point $P$ in the fixed frame, and $\boldsymbol{R}$ is a $3 \times 3$ rotation matrix providing the orientation of $P X^{\prime} Y^{\prime} Z^{\prime}$ relative to $O X Y Z$. However, the entries of $\boldsymbol{R}$ are not independent, since they must define an orthogonal matrix of positive determinant. Such a constraint can be defined in a variety of ways, e.g. by establishing appropriate dotand cross-product equations on the columns of $\boldsymbol{R}$, but more intuitive representations of the orientation are obtained when three-parameter expressions for $\boldsymbol{R}$ are adopted. Due to its attractive properties, we will use here the parameterization provided by tilt-and-torsion angles [16], $\tau=\{\phi, \theta, \sigma\}$, for which

$$
\boldsymbol{R}=\boldsymbol{R}_{z}(\phi) \boldsymbol{R}_{y}(\theta) \boldsymbol{R}_{z}(\sigma-\phi),
$$

but any other parameterization could be used if desired.

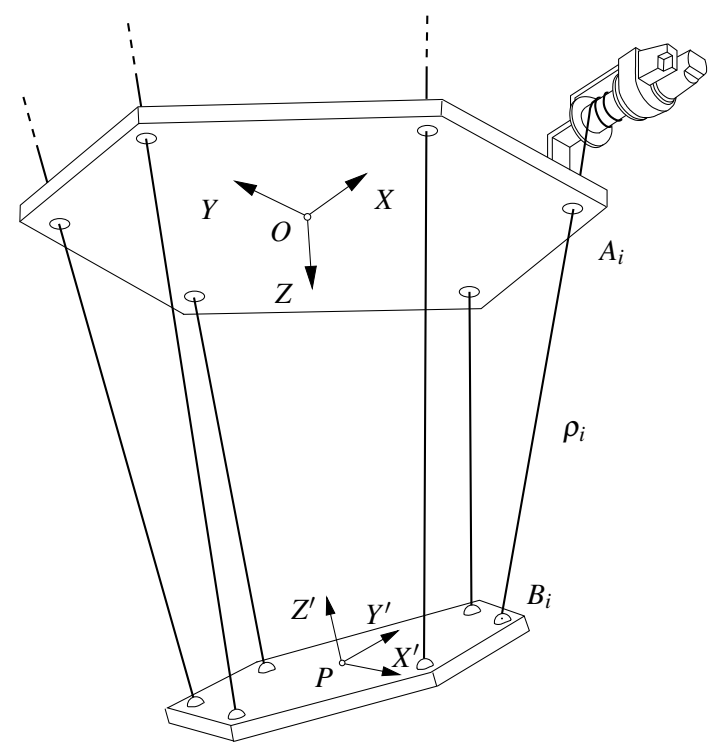

Fig. 1 A cable-driven hexapod. The platform is maintained under a stable position due to the action of gravity. 
In practice, not all configurations can be reached, because the cable lengths $\rho_{i}$ are constrained to lie within the range $\left(\rho_{i}, \overline{\rho_{i}}\right)$ of allowable values, with $\rho_{i}>0$. Thus, if $\boldsymbol{a}_{i}$ and $\boldsymbol{b}_{i}$ denote the position vectors of the anchor points $A_{i}$ and $B_{i}$ of the $i$ th leg (Fig. 1), expressed in $O X Y Z$ and $P X^{\prime} Y^{\prime} Z^{\prime}$ respectively, and $\boldsymbol{u}_{i}$ is the vector $B_{i}-A_{i}$ expressed in $O X Y Z$, the configuration will only be valid if it satisfies

$$
\begin{array}{r}
\boldsymbol{p}+\boldsymbol{R} \boldsymbol{b}_{i}-\boldsymbol{u}_{i}-\boldsymbol{a}_{i}=0, \\
\boldsymbol{u}_{i}^{\top} \boldsymbol{u}_{i}-\rho_{i}^{2}=0
\end{array}
$$

with

$$
\rho_{i} \in\left(\underline{\rho_{i}}, \overline{\rho_{i}}\right)
$$

for $i=1, \ldots, 6$.

Additionally, any configuration must be wrench-feasible, in the sense that it must allow the platform to equilibrate any external wrench $\hat{\boldsymbol{w}}$ acting on it, subject to lie inside a prescribed six-dimensional region $\mathscr{W} \subset \mathbb{R}^{6}$. The significance of $\mathscr{W}$ depends on the particular context of application but, typically, $\mathscr{W}$ is determined by the gravitational wrench $\hat{\boldsymbol{w}}_{0}$ acting on the platform, and by bounds on the perturbations introduced by inertia forces or external agents like the wind, for instance. Specifically, the wrench-feasibility requirement on a given $\boldsymbol{q}$ implies that for each wrench $\hat{\boldsymbol{w}} \in \mathscr{W}$ there must be a vector

$$
\boldsymbol{f}=\left[f_{1}, \ldots, f_{6}\right]^{\top} \in \mathscr{D}=\left(\underline{f_{1}}, \overline{f_{1}}\right) \times \ldots \times\left(\underline{f_{6}}, \overline{f_{6}}\right)
$$

of cable tensions satisfying

$$
\boldsymbol{J}(\boldsymbol{q}) \cdot \boldsymbol{f}=\hat{\boldsymbol{w}},
$$

where $\boldsymbol{J}(\boldsymbol{q})$ is the $6 \times 6$ screw Jacobian of the manipulator at $\boldsymbol{q}$, and $\left(f_{i}, \bar{f}_{i}\right)$ is the range of cable tensions that can be resisted by the $i$ th cable, with $f_{i}>0$. Here, $\hat{\boldsymbol{w}}, \hat{\boldsymbol{w}}_{0}$, and $\boldsymbol{J}(\boldsymbol{q})$ will be assumed to be given in a frame $P X Y Z$ centered in $P$ and parallel to $O X Y Z$, although any other frame could be assumed if desired, and $\mathscr{W}$ will be a six-dimensional ellipsoid defined as

$$
\left(\hat{\boldsymbol{w}}-\hat{\boldsymbol{w}}_{0}\right)^{\top} \boldsymbol{E}\left(\hat{\boldsymbol{w}}-\hat{\boldsymbol{w}}_{0}\right) \leq 1,
$$

where $\boldsymbol{E}$ is a constant $6 \times 6$ positive-definite symmetric matrix.

Now, let us define the wrench-feasible $C$-space of the manipulator, $\mathscr{C}$, as the set of $\boldsymbol{q} \in S E(3)$ for which the platform is able to counteract all $\hat{\boldsymbol{w}} \in \mathscr{W}$ with $\rho_{i} \in\left(\rho_{i}, \overline{\rho_{i}}\right)$ and $\boldsymbol{f} \in \mathscr{D}$. Given two configurations in $\mathscr{C}, \boldsymbol{q}_{1}$ and $\boldsymbol{q}_{2}$, the goal of this paper is to provide an algorithm for computing a path on $\mathscr{C}$ connecting them, if one exists, or to determine path non-existence otherwise. To this end, we next define a system of equations that are suitable to compute such path using a continuation approach. 


\section{Equations of the wrench-feasible C-space}

Let $\boldsymbol{f}_{0}$ be the vector of cable tensions corresponding to a given $\hat{\boldsymbol{w}}_{0} \in \mathscr{W}$, i.e.,

$$
J(q) \cdot f_{0}=\hat{w}_{0} .
$$

By noting that $\boldsymbol{J}(\boldsymbol{q})\left(\boldsymbol{f}-\boldsymbol{f}_{0}\right)=\hat{\boldsymbol{w}}-\hat{\boldsymbol{w}}_{0}$, it is easy to see that, for a given $\boldsymbol{q}$, the set $\mathscr{F}(\boldsymbol{q})$ of cable tensions $\boldsymbol{f}$ corresponding to all $\hat{\boldsymbol{w}} \in \mathscr{W}$ is the ellipsoid given by

$$
\left(\boldsymbol{f}-\boldsymbol{f}_{0}\right)^{\top} \boldsymbol{B}\left(\boldsymbol{f}-\boldsymbol{f}_{0}\right) \leq 1,
$$

where $\boldsymbol{B}=\boldsymbol{J}(\boldsymbol{q})^{\top} \boldsymbol{E} \boldsymbol{J}(\boldsymbol{q})$. This ellipsoid will be bounded in all directions or unbounded in some, depending on whether $\operatorname{det}(\boldsymbol{J}(\boldsymbol{q})) \neq 0$ or not. However, it is not difficult to see that that $\boldsymbol{J}(\boldsymbol{q})$ is non-singular for all $\boldsymbol{q} \in \mathscr{C}$ (Appendix A), so that $\mathscr{F}(\boldsymbol{q})$ will always be a bounded ellipsoid in our case.

Now, for $\boldsymbol{q}$ to be wrench-feasible, we must have $\mathscr{F}(\boldsymbol{q}) \subseteq \mathscr{D}$, which can be checked as follows. For each $i=1, \ldots, 6$ let $\boldsymbol{v}_{i} \in \mathbb{R}^{6}$ be a vector satisfying

$$
\left.\begin{array}{c}
\boldsymbol{v}_{i}^{\top} \boldsymbol{B} \boldsymbol{v}_{i}=1 \\
\boldsymbol{B}^{i} \boldsymbol{v}_{i}=\mathbf{0}
\end{array}\right\}
$$

where $\boldsymbol{B}^{i}$ stands for the matrix $\boldsymbol{B}$ with its $i$ th row removed. Observe that if $\boldsymbol{J}(\boldsymbol{q})$ is non-singular, then $\boldsymbol{B}$ and $\boldsymbol{B}^{i}$ are full row rank, and if the $i$ th component of $\boldsymbol{v}_{i}$, $v_{i, i}$, is chosen non-negative, then there is exactly one vector $\boldsymbol{v}_{i}$ satisfying Eq. (5). Using Lagrange multipliers, it can be shown that, for the solutions $\boldsymbol{v}_{i}$ of Eq. (5) with $v_{i, i} \geq 0, \boldsymbol{f}_{0}-\boldsymbol{v}_{i}$ and $\boldsymbol{f}_{0}+\boldsymbol{v}_{i}$ are the vectors in $\mathscr{F}(\boldsymbol{q})$ attaining the smallest and largest value along the $i$ th coordinate. Hence, when $\operatorname{det}(\boldsymbol{J}(\boldsymbol{q})) \neq 0, \mathscr{F}(\boldsymbol{q}) \subseteq \mathscr{D}$ if, and only if,

$$
f_{0, i}-v_{i, i}>\underline{f_{i}}
$$

and

$$
f_{0, i}+v_{i, i}<\overline{f_{i}},
$$

for $i=1, \ldots, 6$. These two conditions are equivalent to imposing

$$
\begin{aligned}
& \left(f_{0, i}-v_{i, i}-\underline{f_{i}}\right) \cdot s_{i}=1, \\
& \left(\overline{f_{i}}-f_{0, i}-v_{i, i}\right) \cdot t_{i}=1,
\end{aligned}
$$

together with the inequalities $s_{i} \geq 0$, and $t_{i} \geq 0$, where $s_{i}$ and $t_{i}$ are newly-defined auxiliary variables. Looking at the first equation, for instance, it is clear that neither $f_{0, i}-v_{i, i}-f_{i}$ nor $s_{i}$ can be zero, so that for any $s_{i} \geq 0$, it will always be $f_{0, i}-v_{i, i}>\underline{f_{i}}$ as desired. In a similar way, the cable-length constraints $\rho_{i} \in\left(\underline{\rho_{i}}, \overline{\rho_{i}}\right)$ are equivalent to imposing

$$
\left(\rho_{i}-\underline{\rho_{i}}\right) \cdot\left(\overline{\rho_{i}}-\rho_{i}\right) \cdot g_{i}=1,
$$

in conjuntion with the inequality constraint $g_{i} \geq 0$ acting on the new variable $g_{i}$. 
At this point, let us consider the system formed by Eqs. (1)-(8), which we will write compactly as

$$
\boldsymbol{F}(\boldsymbol{x})=\mathbf{0},
$$

where $\boldsymbol{x}$ refers to an $n_{x}$-vector encompassing all of its variables, and let us distinguish between the solution set of Eq. (9),

$$
\mathscr{M}=\{\boldsymbol{x}: \boldsymbol{F}(\boldsymbol{x})=\mathbf{0}\},
$$

and the set

$$
\mathscr{M}^{+}=\left\{\boldsymbol{x} \in \mathscr{M}: v_{i, i} \geq 0, s_{i} \geq 0, t_{i} \geq 0, g_{i} \geq 0, \text { for } i=1, \ldots, 6\right\} .
$$

Note that every configuration $\boldsymbol{q} \in \mathscr{C}$ has a corresponding point $\boldsymbol{x} \in \mathscr{M}^{+}$and, conversely, each point in $\mathscr{M}^{+}$projects down to one $\boldsymbol{q} \in \mathscr{C}$. Moreover, any continuous path in $\mathscr{C}$ will also be represented by a continuous path in $\mathscr{M}^{+}$and viceversa, so that the original problem of computing a wrench-feasible path in $\mathscr{C}$ from $\boldsymbol{q}_{1}$ to $\boldsymbol{q}_{2}$ can be reduced to that of computing a path in $\mathscr{M}^{+}$connecting points $\boldsymbol{x}_{1}$ and $\boldsymbol{x}_{2}$ corresponding to $\boldsymbol{q}_{1}$ to $\boldsymbol{q}_{2}$.

Two properties can be exploited regarding the structure of $\mathscr{M}$ and $\mathscr{M}^{+}$(Appendix A), which allow to apply a continuation strategy to connect $\boldsymbol{x}_{1}$ and $\boldsymbol{x}_{2}$ using Eq. (9). It is easy to see, first, that $v_{i, i}, s_{i}, t_{i}$, and $g_{i}$ never vanish on $\mathscr{M}$, so that there does not exist any path traversing from $\mathscr{M}^{+}$to its complement $\mathscr{M} \backslash \mathscr{M}^{+}$. Thus, if $\boldsymbol{x}_{1}$ and $\boldsymbol{x}_{2}$ are chosen with positive values for $v_{i, i}, s_{i}, t_{i}$, and $g_{i}$, then any continuous path on $\mathscr{M}$ connecting $\boldsymbol{x}_{1}$ and $\boldsymbol{x}_{2}$ will entirely lie on $\mathscr{M}^{+}$, and it will correspond to a path on $\mathscr{C}$ therefore. In other words, when trying to connect $\boldsymbol{x}_{1}$ and $\boldsymbol{x}_{2}$ by continuation on $\mathscr{M}$, the positivity constraints on $v_{i, i}, s_{i}, t_{i}$, and $g_{i}$ will be implicitly fulfilled, and they can be safely neglected. Second, it can be shown that $\mathscr{M}$, and in particular $\mathscr{M}^{+}$, is a six-dimensional smooth manifold everywhere, so that every point $\boldsymbol{x}$ has a well-defined tangent space $T_{\boldsymbol{x}} \mathscr{M}$, which facilitates the application of the following continuation strategy to connect $\boldsymbol{x}_{1}$ and $\boldsymbol{x}_{2}$, because no bifurcations, sharpnesses, or dimension changes will be found when traversing $\mathscr{M}$.

\section{Exploring $\mathscr{M}$ for a connecting path}

To determine a path on $\mathscr{M}$ connecting two points $\boldsymbol{x}_{1}$ and $\boldsymbol{x}_{2}$ we can gradually construct an atlas of $\mathscr{M}$, i.e., a collection of charts where each chart $C_{i}$ defines a local map from a domain $\mathscr{P}_{i} \subset \mathbb{R}^{6}$ to an open set of $\mathscr{M}$ around a point $\boldsymbol{x}_{i} \in \mathscr{M}$, initially $\boldsymbol{x}_{1}$. The atlas will be computed using the higher-dimensional continuation approach proposed in [14], which defines the local map for chart $C_{i}$ using $\boldsymbol{\Psi}_{i}$, an orthonormal basis of $T_{\boldsymbol{x}_{i}} \mathscr{M}$. The map is defined by first selecting a vector $\boldsymbol{u}_{j}^{i} \in \mathbb{R}^{6}$ of parameters (Fig. 2, left), which is used to generate a point $\boldsymbol{x}_{j}^{i} \in \mathbb{R}^{n_{x}}$ in the neighborhood of $\boldsymbol{x}_{i}$, using $\boldsymbol{x}_{j}^{i}=\boldsymbol{x}_{i}+\boldsymbol{\Psi}_{i} \boldsymbol{u}_{j}^{i}$. Then, a point $\boldsymbol{x}_{j} \in \mathscr{M}$ corresponding to the projection of $\boldsymbol{x}_{j}^{i}$ on 

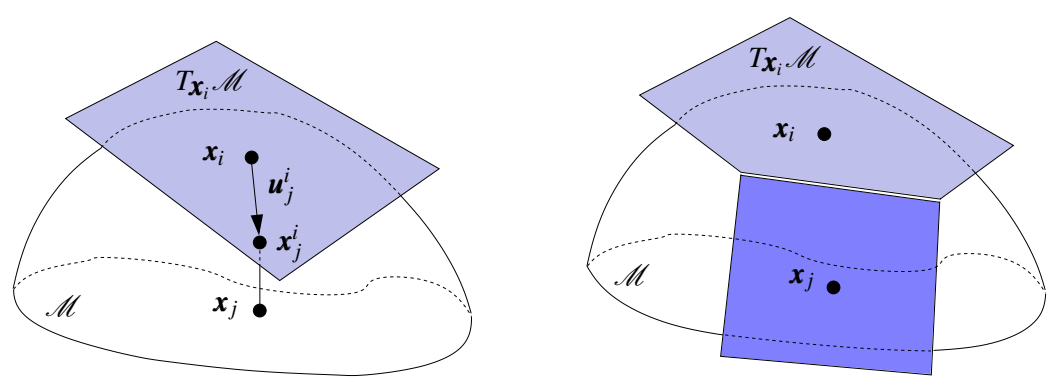

Fig. 2 The higher-dimensional continuation method applied to a 2-dimensional manifold in $\mathbb{R}^{3}$.
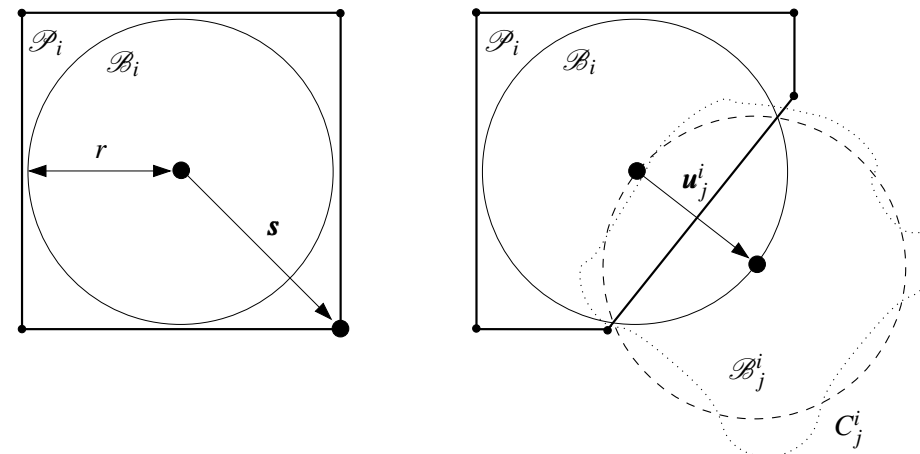

Fig. 3 The process of chart construction.

$\mathscr{M}$ is computed, by solving the system formed by $\boldsymbol{F}\left(\boldsymbol{x}_{j}\right)=\mathbf{0}$ and $\boldsymbol{\Psi}_{i}^{\top}\left(\boldsymbol{x}_{j}-\boldsymbol{x}_{j}^{i}\right)=\mathbf{0}$ using a Newton method initialized at $\boldsymbol{x}_{j}^{i}$.

Each point $\boldsymbol{x}_{j}$ is the potential center of a new chart (Fig. 2, right), and a method due to Henderson can be used to decide where to place the chart centers so as to ensure a good coverage of the manifold [14]. In his approach, the domain $\mathscr{P}_{i}$ of chart $C_{i}$ is initialized as a 6-dimensional hypercube enclosing a ball $\mathscr{B}_{i}$ of radius $r$, both defined in $T_{\boldsymbol{x}_{i}} \mathscr{M}$, as illustrated in Fig. 3, left. A vertex of $\mathscr{P}_{i}$ exterior to $\mathscr{B}_{i}$, with position vector $\boldsymbol{s}$, is used to generate a point $\boldsymbol{x}_{j}^{i}$, with $\boldsymbol{u}_{j}^{i}=\alpha \cdot \boldsymbol{s} /\|\boldsymbol{s}\|$, where $\alpha$ is initialized to $r$. If the projection of $\boldsymbol{x}_{j}^{i}$ to $\mathscr{M}$ does not converge, or if the new chart $C_{j}$ at $\boldsymbol{x}_{j}$ is too far or too different from $C_{i}$, the new chart is discarded and a new attempt of chart generation is performed with a smaller $\alpha$, allowing to adapt the size of the area covered by each chart to the local curvature of the manifold. When $C_{j}$ is valid, it is used to crop $\mathscr{P}_{i}$ from the intersection between $\mathscr{B}_{i}$ and $C_{j}^{i}$, the projection on $T_{\boldsymbol{x}_{i}} \mathscr{M}$ of the part of the manifold covered by $C_{j}$. This projection is approximated by a ball $\mathscr{B}_{j}^{i}$ of radius $r$ in $T_{\boldsymbol{x}_{i}} \mathscr{M}$, centered at the point given by $\boldsymbol{u}_{j}^{i}$, as shown in Fig. 3, right. The intersection of $\mathscr{B}_{i}$ and $\mathscr{B}_{j}^{i}$ defines a new face for $\mathscr{P}_{i}$ that eliminates some of its vertices (in particular the one given by $\boldsymbol{s}$ ) and generates new ones. Symmetrically, the polytope $\mathscr{P}_{j}$ associated with $C_{j}$ is cropped using $C_{i}$. When 
$C_{i}$ is surrounded by other charts, $\mathscr{P}_{i}$ becomes a convex polytope included in $\mathscr{B}_{i}$, and $C_{i}$ is considered to be closed, meaning that no further expansion of the atlas needs to be attempted from that chart. In practice, as $\mathscr{M}^{+}$is unbounded in the directions $s_{i}, t_{i}$, and $g_{i}$, we impose an upper bound on these variables in order to ensure the termination of the atlas expansion. The charts generated outside these bounds are also considered to be closed. When all charts are closed, the connected component of $\mathscr{M}$ containing the initial point $\boldsymbol{x}_{1}$ gets fully covered. If a path exists from $\boldsymbol{x}_{1}$ to $x_{2}, x_{2}$ must be included in one of the charts of the atlas and, thus, a solution path can be determined by searching on the graph implicitly defined by the chart centers and their neighborhood relations. In practice, however, the expansion of the charts is performed according to an A* search strategy using an admissible heuristic [24], so that the path is returned as soon as it is found without computing the whole atlas, and it is guaranteed to be the shortest possible on $\mathscr{M}$. If $\boldsymbol{x}_{2}$ is not included in any of the charts in the end, path non-existence is established at the considered value for $r$. A detailed analysis of the cost of the algorithm can be found in [21].

\section{Experiments}

The method has been implemented in $\mathrm{C}$, and run on a MacBook Pro equipped with a $2.66 \mathrm{GHz}$ Intel Core $\mathrm{i} 7$ processor. To verify its performance on a realistic situation, the geometric parameters of the NIST Robocane manipulator have been used [1] (Fig. 4). This manipulator follows an octahedral design where both the base and the platform are equilateral triangles of sides $2 b$ and $2 a$, respectively. The fixed reference frame $O X Y Z$ is defined with the $Z$ axis pointing downwards, and a mobile reference frame $P X^{\prime} Y^{\prime} Z^{\prime}$ is attached to the platform. The coordinates of the vertex points of the base, expressed in $O X Y Z$, are $A_{1}=(-b,-b \sqrt{3} / 3,0), A_{2}=$ $(b,-b \sqrt{3} / 3,0)$, and $A_{3}=(0,2 b \sqrt{3} / 3,0)$, and those of the platform, expressed in $P X^{\prime} Y^{\prime} Z^{\prime}$, are $B_{1}=(0,-2 a \sqrt{3} / 3,0), B_{2}=(a, a \sqrt{3} / 3,0)$, and $B_{3}=(-a, a \sqrt{3} / 3,0)$.

In all experiments, the platform is required to withstand a weight of $m$ Newtons applied at a point $P_{m}=\left(\frac{a}{5}, \frac{a}{5}, 0\right)$ in $P X^{\prime} Y^{\prime} Z^{\prime}$. Since the weight always points downwards, this corresponds to a wrench $\hat{\boldsymbol{w}}_{0}=[0,0, m, 0,0,0]^{\top}$ that is constant in a reference frame parallel to $O X Y Z$ translating with $P_{m}$. For simplicity, the small variations that may be introduced by inertia forces or external agents are represented by the ellipsoid $\mathscr{W}$ centered in $\boldsymbol{w}_{0}$ defined by $\boldsymbol{E}=10^{4} \cdot \boldsymbol{I}_{6}$, which is a six-dimensional sphere of radius $10^{-2}$, and the forces and lengths for all cables are set to remain positive but lower than $\overline{f_{i}}=m N$ and $\overline{\rho_{i}}=5 a$, respectively.

To illustrate the complexity of the path planning problem, Fig. 5 shows several slices of the wrench-feasible $\mathrm{C}$-space $\mathscr{C}$ of the manipulator, computed in Matlab using discretization with $a=1, b=2$, and $m=1$. The configurations that cannot be reached due to either cable lengths or forces out of range are represented by the red and blue areas, respectively, while those corresponding to $\mathscr{C}$ are indicated in green. The figure also shows the singularity curves where $\operatorname{det}(\boldsymbol{J}(\boldsymbol{q}))=0$, in red, computed with the method in [3] under no constraints on the cable tensions or lengths. It can 


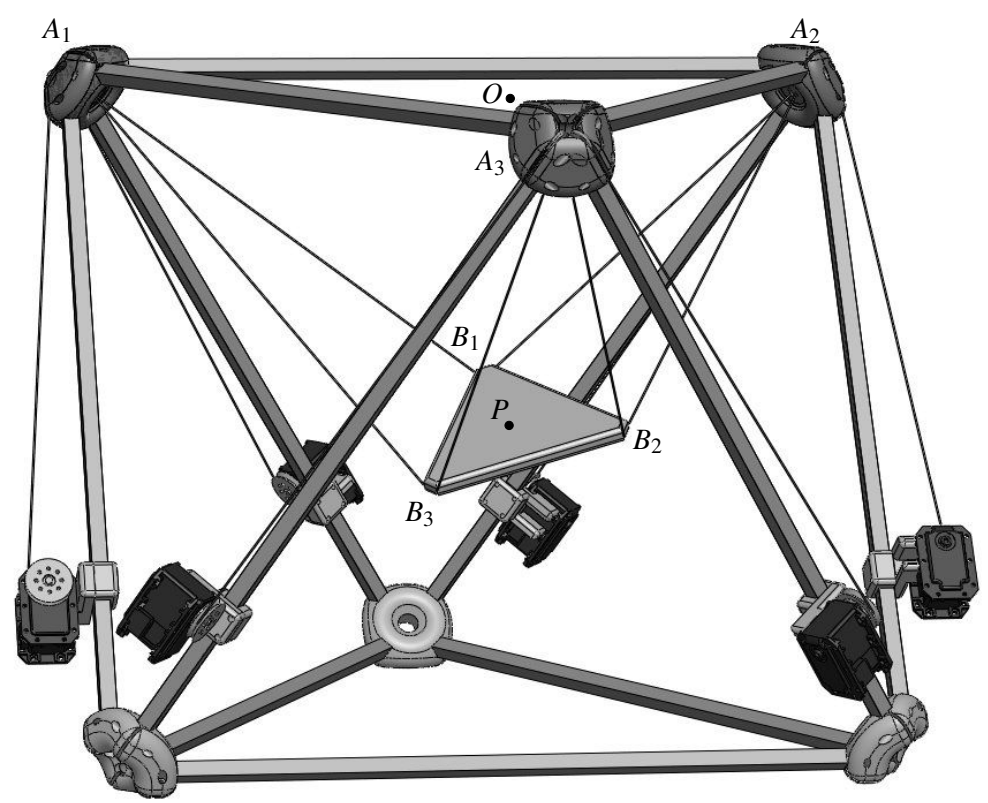

Fig. 4 CAD Model of the octahedral cable-driven hexapod under construction at Institut de Robòtica i Informàtica Industrial (IRI), inspired in the NIST Robocrane manipulator [1] (courtesy of P. Grosch).

be observed that the wrench-feasible C-space naturally avoids crossing singularities, although in some zones this point may not be clear due to the resolution of the discretization, and to the small size of the figures. The top row of Fig. 5 corresponds to slices where the point $P$ and the torsion $\sigma$ are held fixed. In the bottom row, the whole orientation $\tau$ of the platform and one of the coordinates of $P$ are held fixed. As it can be seen from the figures, the navigation between two configurations of $\mathscr{C}$ is not a trivial task. In particular, evaluating the wrench-feasibility conditions on discrete points along a path could result in erroneous paths that, for example, could join points belonging to different connected components of $\mathscr{C}$. The method presented in this paper is able to solve such hard planning queries, even when permitting the variation of all pose parameters. However, due to illustration limitations, the performance of the method is demonstrated here by means of two experiments where four and three pose parameters are held fixed.

In the first experiment, we compute two different paths on the top-center slice of $\mathscr{C}$ shown in Fig. 5, where $\boldsymbol{p}=[0,0,2]^{\top}$ and $\sigma=35^{\circ}$. Using the start $\boldsymbol{q}_{1}$ and goal $\boldsymbol{q}_{2}$ configurations defined by $\boldsymbol{\tau}_{1}=\left\{-0.5,0.9, \frac{7}{36} \pi\right\} \mathrm{rad}$ and $\boldsymbol{\tau}_{2}=\left\{2,0.9, \frac{7}{36} \pi\right\} \mathrm{rad}$, respectively, the resulting path is computed in 20 seconds. Fig. 6 , top, shows this path in red together whith the atlas corresponding to the whole connected component of $\mathscr{C}$ accessible from the start configuration (shown as a green mesh), and the region explored by the algorithm (shaded in grey). In order to evaluate the per- 

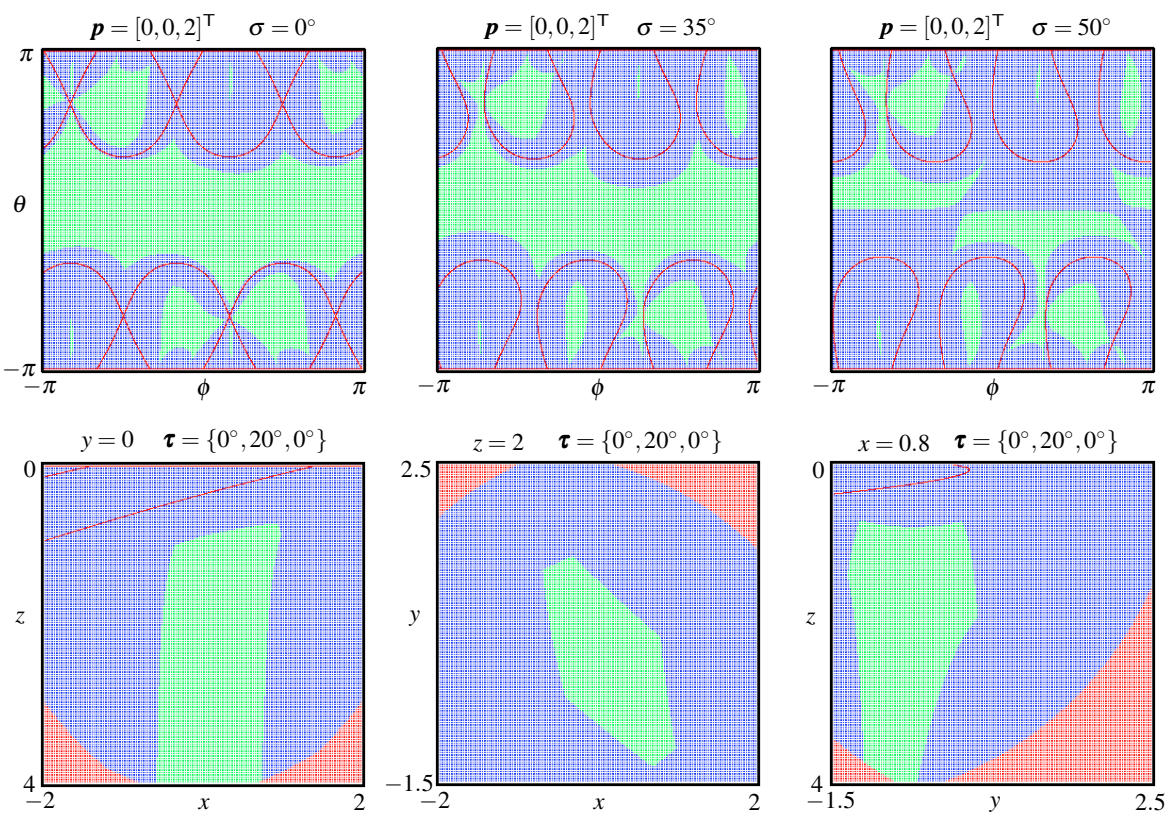

Fig. 5 Slices of the wrench-feasible C-space obtained by discretization. Blue, red and green zones correspond to configurations where some force is out of range, configurations not attainable due to cables length, and configurations belonging to the wrench-feasible C-space, respectively.

formance of the method on a more challenging situation, a second path is computed between configurations $\boldsymbol{q}_{3}$ and $\boldsymbol{q}_{4}$ given by $\boldsymbol{\tau}_{3}=\left\{0.8,-2.4, \frac{7}{36} \pi\right\} \mathrm{rad}$ and $\boldsymbol{\tau}_{4}=\left\{-2.4,2.4, \frac{7}{36} \pi\right\} \mathrm{rad}$. In this case the path is computed in 280 seconds and the region explored is shaded in green (Fig. 6, top). On both planning queries, note that the interpolated path between the start and goal configurations would violate some of the constraints of $\mathscr{C}$, giving rise to uncontrollable motions of the platform, or to breakage of some of the cables, but the computed paths correctly avoid these situations. Indeed, an advantegous property of the continuation strategy employed, which cannot be ensured by methods relying on discretization, is that the computed path will not jump between distinct connected components of $\mathscr{C}$, even when such components are close to each other, thus ensuring that the forces on all cables keep within the prescribed ranges along the whole path. As an example, these forces can be seen in Fig. 6, bottom, for the path from $\boldsymbol{q}_{3}$ to $\boldsymbol{q}_{4}$. The evolution along this path of the maximum and minimum tensions on each cable are plotted in green and blue, respectively. Towards the end of the path, some cable may attain a near-zero tension, but this can be easily avoided if desired, by simply setting a higher value of $f_{i}$.

On the second experiment, only three pose variables are held fixed, namely $x=0, y=0$, and $\sigma=35^{\circ}$, giving rise to a three-dimensional slice of the wrenchfeasible C-space $\mathscr{C}$. Here, the start and goal configurations, $\boldsymbol{q}_{5}$ and $\boldsymbol{q}_{6}$, are given by $\boldsymbol{p}_{5}=[0,0,1.2]^{\top}, \boldsymbol{\tau}_{5}=\left\{0.8,-2.6, \frac{7}{36} \pi\right\} \mathrm{rad}$ and $\boldsymbol{q}_{6}=\boldsymbol{q}_{4}$. The resulting path can be 


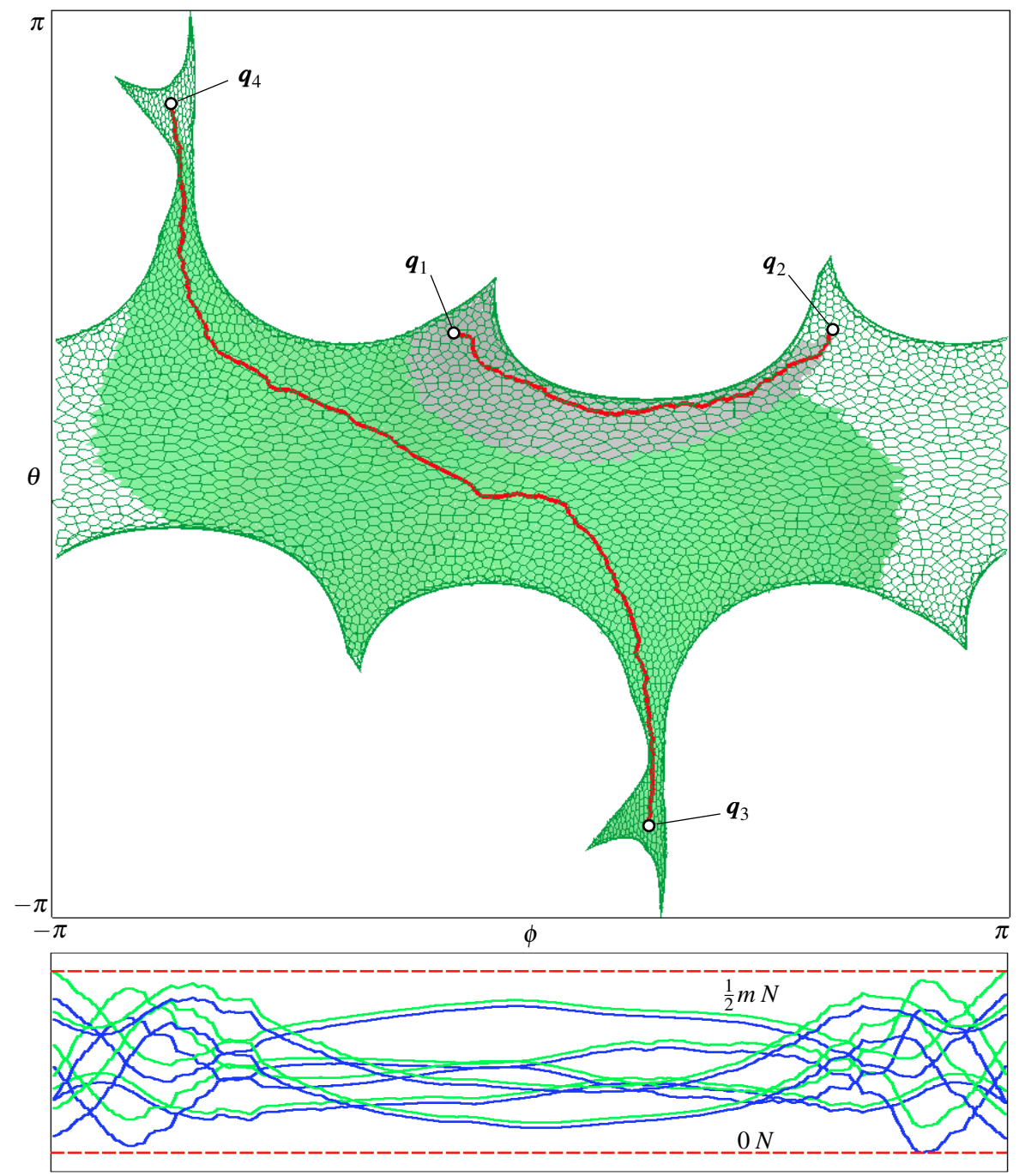

Fig. 6 Top: Two paths and the areas of $\mathscr{C}$ explored to compute them, on the slice of the wrenchfeasible C-space corresponding to $\boldsymbol{p}=[0,0,2]^{\top}$ and $\sigma=35^{\circ}$. Bottom: evolution of the maximum (green) and minimum (blue) possible tensions for each cable, along the path from $\boldsymbol{q}_{3}$ to $\boldsymbol{q}_{4}$.

seen in Fig. 7 in red, together with the atlas generated by the algorithm in green. This case represents a hard planning query and, therefore, the computation time increases significantly. However, once a partial atlas is computed, all planning queries between configurations covered by such atlas can be solved in a few milliseconds. 


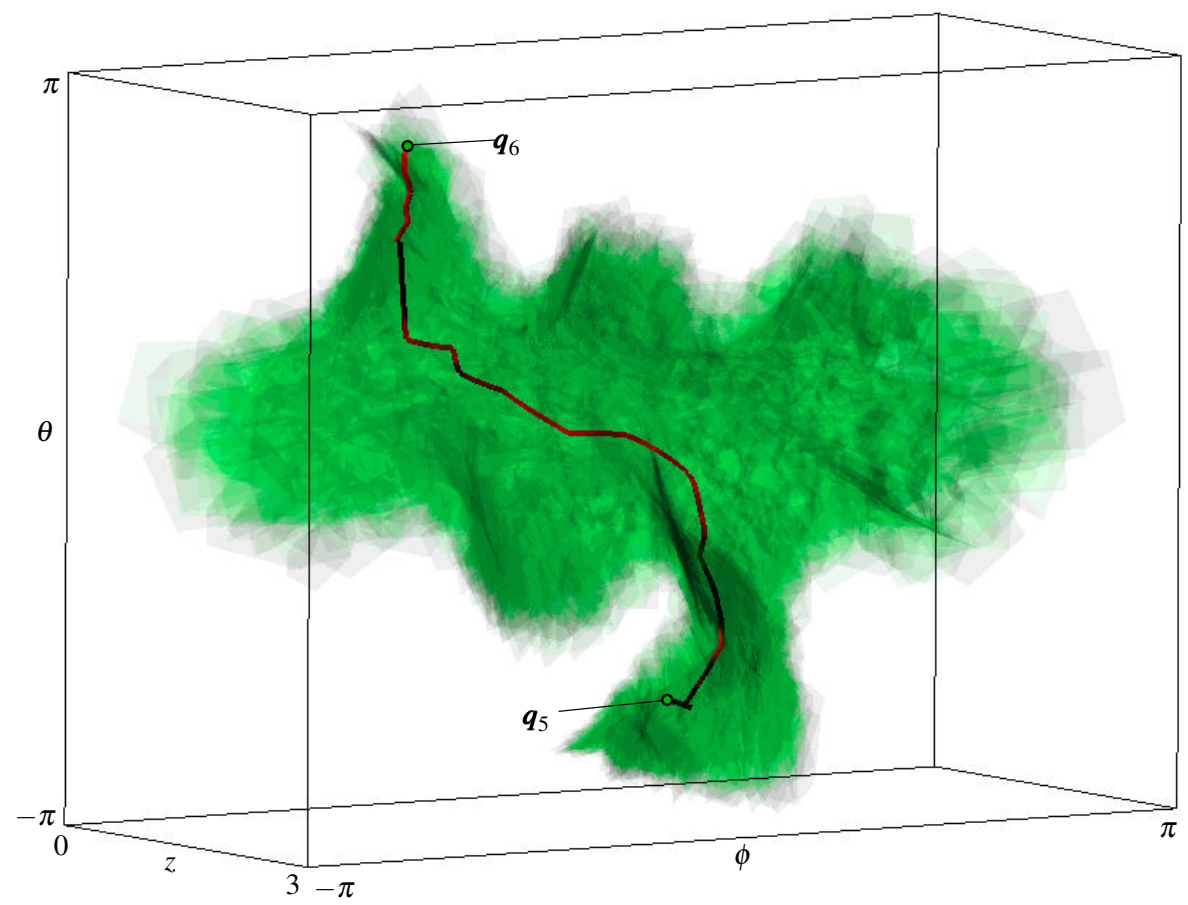

Fig. 7 A path on a three-dimensional slice of the wrench-feasible C-space with $x=0, y=0$ and $\sigma=35^{\circ}$. The green volume corresponds to the portion of $\mathscr{C}$ explored by the algorithm, which is here visualized by projecting the chart polytopes $\mathscr{P}_{i}$ to the space of $z, \phi$, and $\theta$, and representing their faces as semi-transparent walls, in order to visualize the computed path in the interior.

\section{Conclusions and future work}

This paper has presented a path planning method for computing wrench-feasible paths on cable-driven hexapods, i.e., configuration paths that guarantee the resolvability of a six-dimensional set of wrenches at any point on the path. As a by product, the method implicitly ensures that the screw Jacobian of the manipulator will be non-singular along the path, thus allowing a full control of the platform motions at all times. The method has been tested succesfully on several cases of increasing complexity, though only cases where three or four pose variables are held fixed have been shown due to illustration limitations.

The presented approach allows extensions in several possible ways. For example, some additional constraints could be considered, like enforcing a certain degree of positioning accuracy of the platform, or the avoidance of platform collisions (cableplatform or cable-cable collisions, or even those with the environment). While the former constraints can in principle be incorporated using dual developments to those herein presented, the latter require investigating the possibility of randomizing the planner, in the spirit of [8] or [22]. 


\section{Acknowledgements}

This work has been partially funded by the Spanish Government under contract DPI2010-18449, and by a Juan de la Cierva contract supporting the first author. The authors would also like to thank Patrick Grosch for his intensive work on the prototype shown in Fig. 4.

\section{Appendix A: Properties of $\mathscr{M}$}

This appendix is devoted to the proof of two properties that are essential in order to apply the continuation strategy described in Section 4. The first one is the non-nullity of $s_{i}, t_{i}, g_{i}$ and $v_{i, i}$, and the second one is the smoothness of the sixdimensional manifold $\mathscr{M}$. A by-product of the second property is the fact that $\boldsymbol{J}(\boldsymbol{q})$ is non-singular for all $\boldsymbol{q} \in \mathscr{C}$.

From Eqs. (6), (7), and (8) it follows directly that $s_{i}, t_{i}$ and $g_{i}$ can never be zero on $\mathscr{M}$. The same property for $v_{i, i}$ can be proved by contradiction. Let us assume that $v_{i, i}=0$ for some $i$. If we consider Eq. (5), then, by replacing $\boldsymbol{B}^{i} \boldsymbol{v}_{i}=\mathbf{0}$ into $\boldsymbol{v}_{i}^{\top} \boldsymbol{B} \boldsymbol{v}_{i}=1$, we obtain the dot product of two vectors: $\boldsymbol{v}_{i}^{\top}$, with $v_{i, i}=0$, and the vector $\boldsymbol{B} \boldsymbol{v}_{i}$, whose components are all zero except that in position $i$. The result of this dot product is 0, which contradicts Eq. (5), as it should be 1. As a result, the set $\mathscr{M}^{+}$and its complement $\mathscr{M} \backslash \mathscr{M}^{+}$are disconnected.

Let us now prove the smoothness of $\mathscr{M}$. If we can verify that $\boldsymbol{F}(\boldsymbol{x})$ is a differentiable function with full rank differential $\boldsymbol{F}_{\boldsymbol{x}}$, then the smoothness of $\mathscr{M}$ will follow from the implicit function theorem. By construction all functions intervening in $\boldsymbol{F}(\boldsymbol{x})$ are differentiable all over $\mathscr{M}$, and the differential matrix $\boldsymbol{F}_{\boldsymbol{x}}$ can be expressed in the following block-triangular form after re-organizing some equations and variables

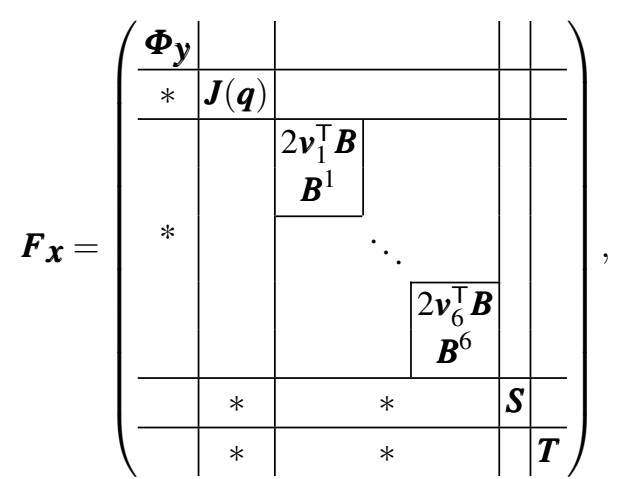

where empty blocks represent zero-matrices and asterisks indicate non-zero blocks. Due to the triangular structure of $\boldsymbol{F}_{\boldsymbol{x}}$ it suffices to verify that the five blocks in the diagonal are full-rank in order to prove the smoothness of $\mathscr{M}$. 
The first block is

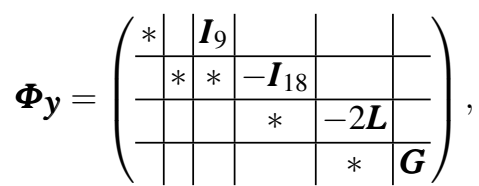

which is the differential matrix of the system $\boldsymbol{\Phi}(\boldsymbol{y})=\mathbf{0}$ formed by Eqs. (1)-(3) and (8) with respect to $\boldsymbol{y}=\left(\boldsymbol{\tau}, \boldsymbol{p}, \boldsymbol{R}, \boldsymbol{u}_{i}, \rho_{i}, g_{i}\right)$. Here $\boldsymbol{L}$ and $\boldsymbol{G}$ are $6 \times 6$ diagonal matrices with diagonal elements $\rho_{i}$ and $\left(\rho_{i}-\rho_{i}\right) \cdot\left(\overline{\rho_{i}}-\rho_{i}\right)$, respectively. To see that $\boldsymbol{\Phi}_{\boldsymbol{y}}$ is full rank, observe that its last four block-columns comprise a non-singular square submatrix of maximum size, as its diagonal elements do not vanish over $\mathscr{M}$ by virtue of Eq. (8) and the fact that $\rho_{i}>0$.

The remaining four diagonal blocks of $\boldsymbol{F}_{\boldsymbol{x}}$ are the differential matrices of Eqs. (4)-(7) with respect to the variables $f_{0}, v_{i}, s_{i}$ and $t_{i}$, respectively, where the blocks $\boldsymbol{S}$ and $\boldsymbol{T}$ are $6 \times 6$ diagonal matrices with elements $f_{0, i}-v_{i, i}-f_{i}$ and $\overline{f_{i}}-f_{0, i}-v_{i, i}$, respectively. The screw Jacobian $\boldsymbol{J}(\boldsymbol{q})$ can be shown to be full rank over $\mathscr{M}$ by contradiction. Indeed, if $\boldsymbol{J}\left(\boldsymbol{q}_{s}\right)$ were rank deficient for some $\boldsymbol{q}_{s}$, then so would be $\boldsymbol{B}$, and therefore $\operatorname{ker} \boldsymbol{B}$ would contain non-zero vectors. In such case, for some $i$ all solutions of $\boldsymbol{B}^{i} \boldsymbol{v}_{i}=0$ would satisfy $\boldsymbol{v}_{i} \in \operatorname{ker} \boldsymbol{B}$ and, thus, it would be $\boldsymbol{v}_{i}^{\top} \boldsymbol{B} \boldsymbol{v}_{i}=0$, which contradicts Eq. (5) and, hence, $\boldsymbol{J}(\boldsymbol{q})$ cannot be rank deficient over $\mathscr{M}$. The $6 \times 6$ block matrices involving $\boldsymbol{B}$ and $\boldsymbol{B}^{i}$ can only be rank deficient if $v_{i, i}=0$, but this can never happen as we have already seen. All these blocks are therefore full rank over $\mathscr{M}$. Finally, it is clear that $\boldsymbol{S}$ and $\boldsymbol{T}$ are also full rank over $\mathscr{M}$, since their diagonal elements never vanish due to Eqs. (6) and (7), and this completes the proof of the smoothness of $\mathscr{M}$ and, in particular, that of $\mathscr{M}^{+}$.

It is worth mentioning that not only $\mathscr{M}$ and $\mathscr{M}^{+}$are smooth, but also any slice taken as a combination of the angular and position parameters, $\boldsymbol{\tau}$ and $\boldsymbol{p}$. Indeed, taking any of these slices implies only the removal of some columns amongst the first two blocks of $\boldsymbol{\Phi}_{\boldsymbol{y}}$, which does not change the global rank of the differential $\boldsymbol{F} \boldsymbol{x}$ corresponding to the considered slice.

\section{References}

[1] Albus, J., Bostelman, R., Dagalakis, N.: The NIST Robocrane. Journal of Robotic Systems 10(5), 709-724 (1993)

[2] Bhattacharya, S., Hatwal, H., Ghosh, A.: Comparison of an exact and an approximate method of singularity avoidance in platform type parallel manipulators. Mechanism and Machine Theory 33(7), 965-974 (1998)

[3] Bohigas, O., Zlatanov, D., Ros, L., Manubens, M., Porta, J.: Numerical computation of manipulator singularities. In: Proc. of the IEEE Int. Conf. on Robotics and Automation (2012)

[4] Borgstrom, P., Jordan, B., Borgstrom, B., Stealey, M., Sukhatme, G., Batalin, M., Kaiser, W.: Nims-pl: A cable-driven robot with self-calibration capabili- 
ties. Robotics, IEEE Transactions on 25(5), 1005 -1015 (2009)

[5] Bosscher, P., II, R.L.W., Bryson, L.S., Castro-Lacouture, D.: Cable-suspended robotic contour crafting system. Automation in Construction 17, 45-55 (2007)

[6] Bosscher, P., Riechel, A., Ebert-Uphoff, I.: Wrench-feasible workspace generation for cable-driven robots. IEEE Trans. on Robotics 22(5), 890-902 (2006)

[7] Cheng, P., Fink, J., Kim, S., Kumar, V.: Cooperative towing with multiple robots. In: G. Chirikjian, H. Choset, M. Morales, T. Murphey (eds.) Algorithmic Foundation of Robotics VIII, Springer Tracts in Advanced Robotics, vol. 57, pp. 101-116. Springer Berlin / Heidelberg (2009)

[8] Cortés, J., Siméon, T.: Probabilistic motion planning for parallel mechanisms. In: Proc. of the IEEE Int. Conf. on Rob. and Aut., vol. 3, pp. 4354-4359 (2003)

[9] Dasgupta, B., Mruthyunjaya, T.: Singularity-free path planning for the Stewart platform manipulator. Mechanism and Machine Theory 33(6), 711-725 (1998)

[10] Dash, A.K., Chen, I.M., Yeo, S.H., Yang, G.: Workspace generation and planning singularity-free path for parallel manipulators. Mechanism and Machine Theory 40(7), 776-805 (2005)

[11] Fang, S., Franitza, D., Verhoeven, R., Hiller, M.: Proceedings of the 11th IFToMM world congress in mechanism and machine science. China Machinery Press, Tianjin, China (2003)

[12] Gouttefarde, M., Daney, D., Merlet, J.P.: Interval-analysis-based determination of the wrench-feasible workspace of parallel cable-driven robots. IEEE Transactions on Robotics 27(1), 1-13 (2011)

[13] Harmon, T., Ambrose, R., Gilbert, R., Fisher, J., Stealey, M., Kaiser, W.: Highresolution river hydraulic and water quality characterization using rapidly deployable networked infomechanical systems (NIMS RD). Environmental Engineering Science 24(2), 151-159 (2007)

[14] Henderson, M.E.: Multiple parameter continuation: Computing implicitly defined $k$-manifolds. Int. J. of Bifurcation and Chaos 12(3), 451-476 (2002)

[15] Hiller, M., Fang, S., Mielczarek, S., Verhoeven, R., Franitza, D.: Design, analysis and realization of tendon-based parallel manipulators. Mechanism and Machine Theory 40(4), 429-445 (2005)

[16] I.A. Bonev, D.Z., Gosselin, C.: Advantages of the modified Euler angles in the design and control of PKMs. In: Parallel Kinematic Machines International Conference, Chemnitz, Germany, April 23-25, pp. 171-188 (2002)

[17] Lahouar, S., Ottaviano, E., Zeghoul, S., Romdhane, L., Ceccarelli, M.: Collision free path-planning for cable-driven parallel robots. Robotics and $\mathrm{Au}-$ tonomous Systems 57(11), 1083-1093 (2009)

[18] Lytle, A.M., Saidi, K.S., Bostelman, R.V., Stone, W.C., Scott, N.A.: Adapting a teleoperated device for autonomous control using three-dimensional positioning sensors: experiences with the NIST RoboCrane. Automation in Construction 13, 101-118 (2004)

[19] Merlet, J.P., Daney, D.: A portable, modular parallel wire crane for rescue operations. In: Robotics and Automation (ICRA), 2010 IEEE International Conference on, pp. $2834-2839$ (2010) 
[20] Oh, S.R., Ryu, J.C., Agrawal, S.K.: Dynamics and control of a helicopter carrying a payload using a cable-suspended robot. Journal of Mechanical Design 128(5), 1113-1121 (2006)

[21] Porta, J., Jaillet, L., Bohigas, O.: Randomized path planning on manifolds based on higher-dimensional continuation. The International Journal of Robotics Research 31(2), 201-215 (2012)

[22] Porta, J.M., Jaillet, L.: Path planning on manifolds using randomized higherdimensional continuation. In: D. Hsu, V. Isler, J.C. Latombe, M. Lin (eds.) Algorithmic Foundations of Robotics IX, Springer Tracts in Advanced Robotics, vol. 68, pp. 337-353. Springer (2011)

[23] Pusey, J., Fattah, A., Agrawal, S., Messina, E.: Design and workspace analysis of a 6-6 cable-suspended parallel robot. Mechanism and Machine Theory 3, 761-778 (2004)

[24] Russell, S.J., Norvig, P.: Artificial Intelligence: A Modern Approach. Prentice Hall (2003)

[25] Sen, S., Dasgupta, B., Mallik, A.K.: Variational approach for singularity-free path-planning of parallel manipulators. Mechanism and Machine Theory 38(11), 1165-1183 (2003)

[26] Stump, E., Kumar, V.: Workspaces of cable-actuated parallel manipulators. Journal of Mechanical Design 128(1), 159-167 (2006)

[27] Tadokoro, S., Matsushima, T., Murao, Y., Kohkawa, H.: A parallel cabledriven motion base for virtual acceleration. In: Intelligent Robots and Systems, 2001. Proceedings. 2001 IEEE/RSJ International Conference on, vol. 3, pp. $1700-1705$ (2001)

[28] Verhoeven, R.: Analysis of the workspace of tendon based Stewart platforms. Ph.D. thesis, Universität Duisburg-Essen (2004)

[29] Verhoeven, R., Hiller, M., Tadokoro, S.: Workspace of tendon-driven Stewart platforms: Basics, classification, details on the planar 2-dof class. In: Proceedings of the 4th International Conference on Motion and Vibration Control, pp. 871-876 (1998)

[30] Voglewede, P., Ebert-Uphoff, I.: Overarching framework for measuring closeness to singularities of parallel manipulators. IEEE Trans. on Robotics 21(6), $1037-1045$ (2005) 